\title{
Padronização cirúrgica das abdominoplastias em âncora pós-gastroplastia
}

\author{
Surgical standardization of abdominoplasty after bariatric surgery
}

\author{
Henrique Cardoso \\ TARDelli $^{1}$ \\ Daniel Bacco Vilela ${ }^{2}$ \\ Gabriela Lustri Eiras \\ SCHWARTZMANN $^{3}$ \\ Marcelo Azevedo ${ }^{3}$ \\ Antônio Marcos de Mello \\ JÚNIOR $^{3}$ \\ Jayme Adriano Farina \\ JÚNIOR ${ }^{6}$
}

Trabalho realizado no Hospital das Clínicas da Faculdade de Medicina de Ribeirão Preto da Universidade de São Paulo, Ribeirão Preto, SP, Brasil.

Artigo submetido pelo SGP (Sistema de Gestão de Publicações) da RBCP.

Artigo recebido: 4/4/2011 Artigo aceito: 9/6/2011

\begin{abstract}
RESUMO
Introdução: Os autores propõem alterações na técnica empregada nas dermolipectomias abdominais após cirurgia bariátrica, com o intuito de corrigir imperfeições nos resultados obtidos com a padronização clássica (i.e. ressecção de pele em bloco com manutenção da cicatriz umbilical). Método: Sessenta e sete pacientes ex-obesos foram submetidos à dermolipectomia abdominal em âncora com ressecção parcelada de pele (primeiro o fuso vertical e após o excesso horizontal), confecção de neoumbigo e dermolipectomia pubiana associada. Resultados: A nova padronização adotada permitiu reduzir as insatisfações previamente observadas, com melhor posicionamento da cicatriz horizontal e menor tensão na intersecção das cicatrizes. Conclusão: A nova sistematização facilitou a marcação préoperatória e permitiu refinar alguns aspectos que se mostravam insatisfatórios na experiência com estes casos.
\end{abstract}

Descritores: Obesidade mórbida. Cirurgia Bariátrica. Cirurgia Plástica/métodos. Lipectomia.

\begin{abstract}
Introduction: The authors propose changes in the post-bariatric abdominoplasty technique to address some imperfections (tension on the T-junction, poor placement of the horizontal scar and unaesthetic umbilical scar) seen with the classic standardization (e.g. en-bloc skin resection and maintenance of the umbilicus). Methods: Assessment was realized on 67 patients who had previously been submitted to bariatric surgery and undergone modified vertical abdominoplasty with staged skin resection (first the vertical component and then the horizontal one), neo-onphaloplasty and dermolipectomy of the pubic area. Results: The new standardization led to reduction on the previous described downsides of the technique (better scar positioning and less tension). Conclusions: The new systematization provided an easier pre-operatory marking and improved aesthetic results.
\end{abstract}

Keywords: Morbid obesity. Bariatric surgery. Plastic surgery/methods. Lipectomy.

1. Membro Titular da Sociedade Brasileira de Cirurgia Plástica (SBCP); Médico Assistente da Divisão de Cirurgia Plástica do Hospital das Clínicas da Faculdade de Medicina de Ribeirão Preto da Universidade de São Paulo, Ribeirão Preto, SP, Brasil.

2. Cirurgião Geral; Médico Residente de Cirurgia Plástica do Hospital das Clínicas da Faculdade de Medicina de Ribeirão Preto da Universidade de São Paulo, Ribeirão Preto, SP, Brasil.

3. Membro associado da SBCP; Cirurgião Plástico, Ribeirão Preto, SP, Brasil.

4. Professor Doutor, Membro Titular da SBCP; Chefe da Divisão de Cirurgia Plástica do Hospital das Clínicas da Faculdade de Medicina de Ribeirão Preto da Universidade de São Paulo, Ribeirão Preto, SP, Brasil. 


\section{INTRODUÇÃO}

$\mathrm{O}$ advento dos procedimentos bariátricos criou um novo contingente de pacientes ex-obesos que buscam na Cirurgia Plástica a correção dos grandes excessos cutâneos resultantes da sua grande perda ponderal. Muitos destes pacientes não são adequadamente tratados pelas técnicas convencionais de dermolipectomia abdominal com cicatriz horizontal apenas ${ }^{1}$. Este fato levou ao "renascimento" de técnicas de dermolipectomia em âncora ${ }^{2-5}$, que permitem a ressecção do excesso cutâneo no sentido horizontal e vertical, resultando numa grande cicatriz em "T" invertido (âncora).

A técnica mais comumente descrita na literatura ${ }^{2-8} \mathrm{implica}$ na ressecção em bloco dos componentes vertical e horizontal, mínimo descolamento dos retalhos remanescentes, plicatura da aponeurose do músculo reto do abdome e manutenção da cicatriz umbilical. A sua aplicação, na experiência dos autores, acarretava nas seguintes insatisfações:

- dificuldade na reprodução das marcações pré-operatórias;

- cicatriz horizontal alta e frequentemente com convexidade superior (Figuras 1 e 2);

- pequena distância entre a cicatriz umbilical e a cicatriz horizontal (Figuras 1 e 2);

- tensão excessiva na ponta dos retalhos laterais;

- aspecto estético desfavorável da cicatriz umbilical, geralmente secundário à grande dimensão ou ao alargamento da mesma (Figura 3);

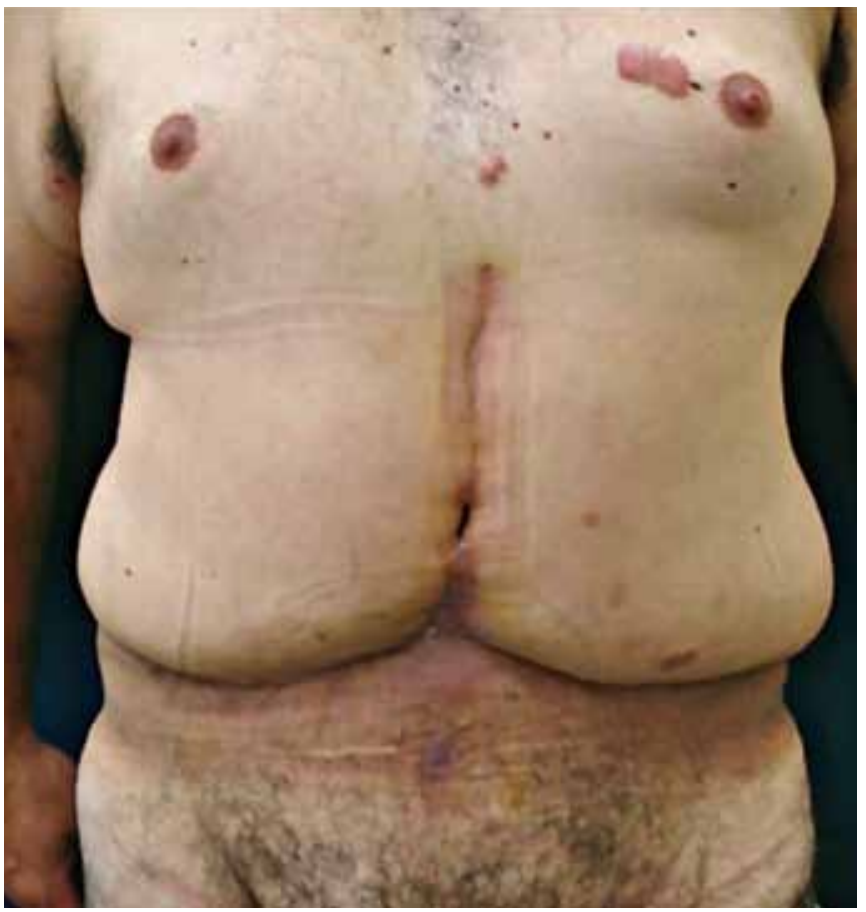

Figura 1 - Paciente em pós-operatório de dermolipectomia em âncora clássica evidenciando cicatriz horizontal alta e com concavidade inferior.

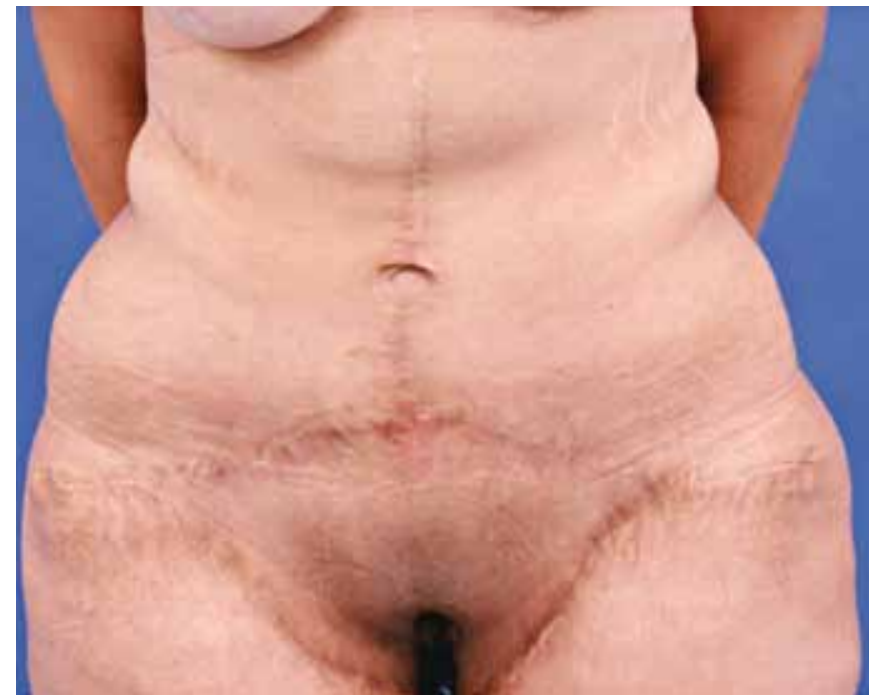

Figura 2 - Paciente em pós-operatório de dermolipectomia em âncora clássica evidenciando cicatriz horizontal alta e com concavidade inferior.

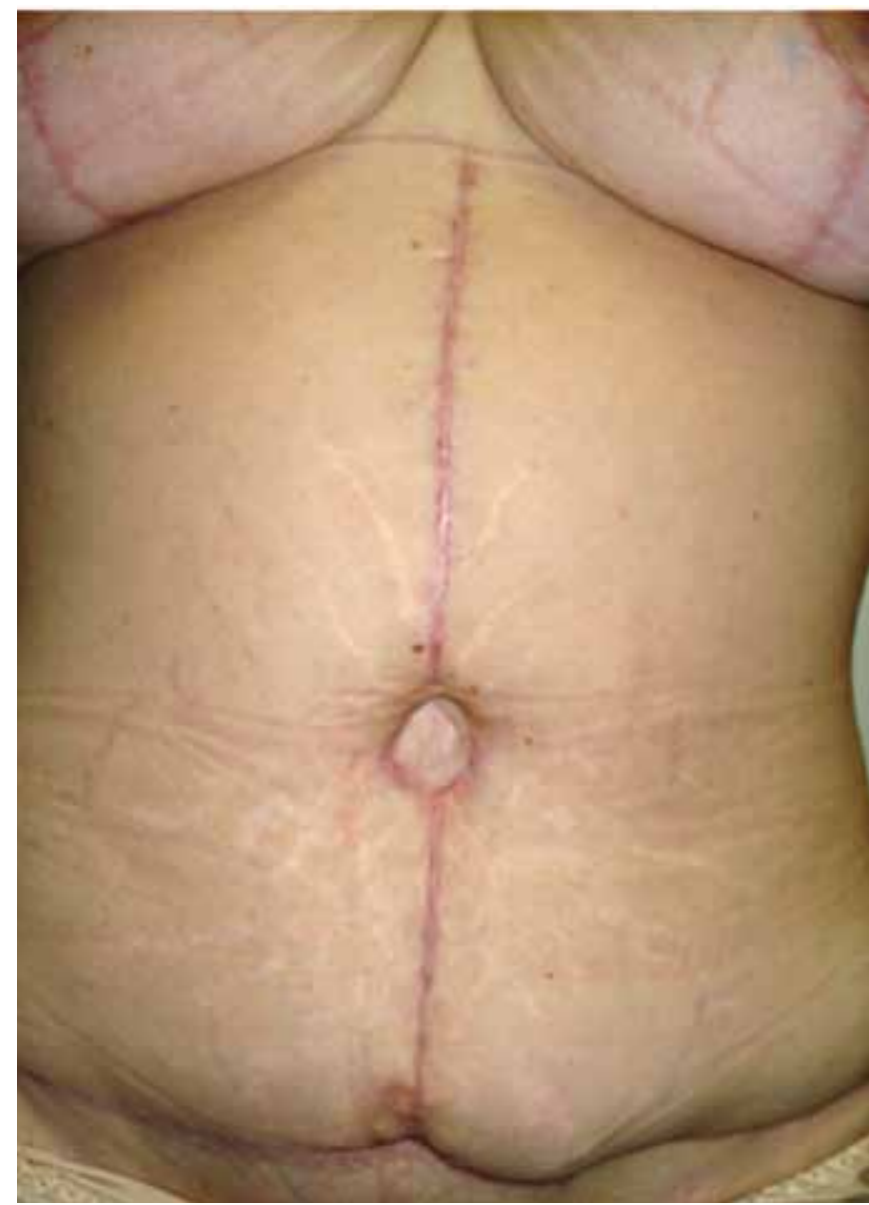

Figura 3 - Cicatriz umbilical de aspecto inestético secundário à distensão da mesma na técnica clássica. 
- excesso cutâneo em região pubiana;

- excesso de pele lateral na porção inferior da cicatriz horizontal.

O presente trabalho tem como intuito descrever alterações adotadas nesta abordagem cirúrgica, a fim de minorar estas insatisfações.

\section{MÉTODO}

Foram avaliados 67 pacientes submetidos à dermolipectomia abdominal em âncora, após grande perda ponderal secundária a procedimento bariátrico, no período de julho de 2006 a dezembro de 2010.

Os critérios de inclusão foram: pacientes operados de cirurgia bariátrica há pelo menos 18 meses, já apresentando estabilização da perda ponderal e liberados pela equipe da Gastrocirurgia, tendo sido submetidos à dermolipectomia abdominal. Não houve exclusão baseada em gênero ou idade.

Os procedimentos bariátricos realizados foram a cirurgia de Capella (gastroplastia vertical e anastomose em "Y" de Roux) em 62 casos e a de Scopinaro (bypass biliopancreático) em 5 casos, sendo todos estes realizados por via aberta com incisão mediana supraumbilical.

A perda ponderal destes pacientes variou de 21 a $96 \mathrm{~kg}$, com média de 55,58 kg, sendo que a média do IMC destes pacientes caiu de $51,5(37,7$ a 68,5$)$ para $30,5(23,1$ a 41,1$)$ após a gastroplastia.

O tempo entre a cirurgia bariátrica e a abdominoplastia variou de 18 a 81 meses, com média de 36 meses. A abdominoplastia foi a primeira intervenção plástica em $91 \%$ destes pacientes. Constatou-se presença de hérnia incisional em 33 pacientes, com necessidade do uso de tela de Marlex ${ }^{\circledR}$ para fechamento da parede em 10 pacientes.

\section{Marcação}

Realiza-se a marcação pré-operatória, inicialmente com o paciente em pé, priorizando a determinação do excesso de pele ao longo da linha média, empregando-se a tensão bimanual na marcação do fuso mediano ou vertical (Figura 4).

Com o paciente agora em decúbito dorsal, confirma-se a simetria das marcações. Em seguida, marca-se a dobra transversal que o paciente apresenta sob a flacidez abdominal no baixo ventre (região suprapúbica) e que norteará a cicatriz horizontal. Para tratar também o excesso de pele pubiana, a marcação da incisão horizontal, nesta área, invade o púbis chegando a 7 ou $8 \mathrm{~cm}$ da rima vulvar (ou da base do pênis nos homens), descrevendo um semicírculo de concavidade superior (Figura 5).

Os retalhos para confecção do neoumbigo são marcados inicialmente com dimensões maiores como prolongamentos de aproximadamente 6 a $7 \mathrm{~cm}$ de largura e $3 \mathrm{~cm}$ de comprimento nas hastes da ressecção mediana, enquanto se traciona

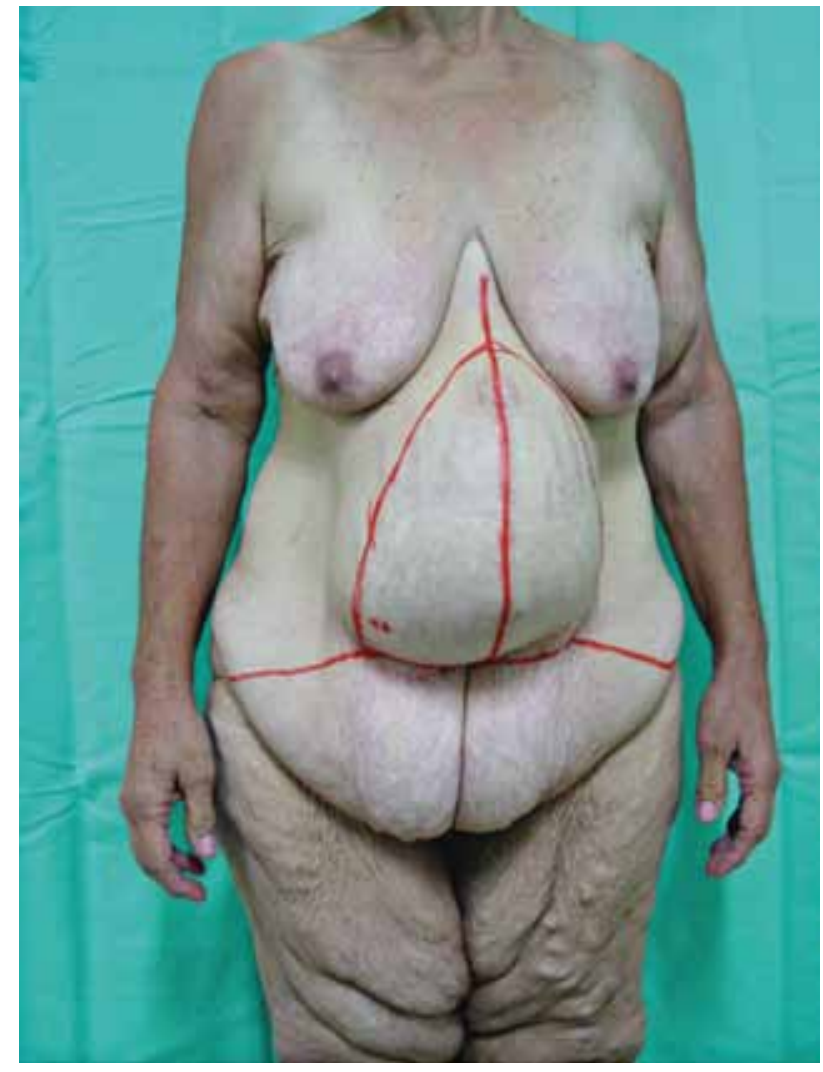

Figura 4-Marcação pré-operatória proposta.

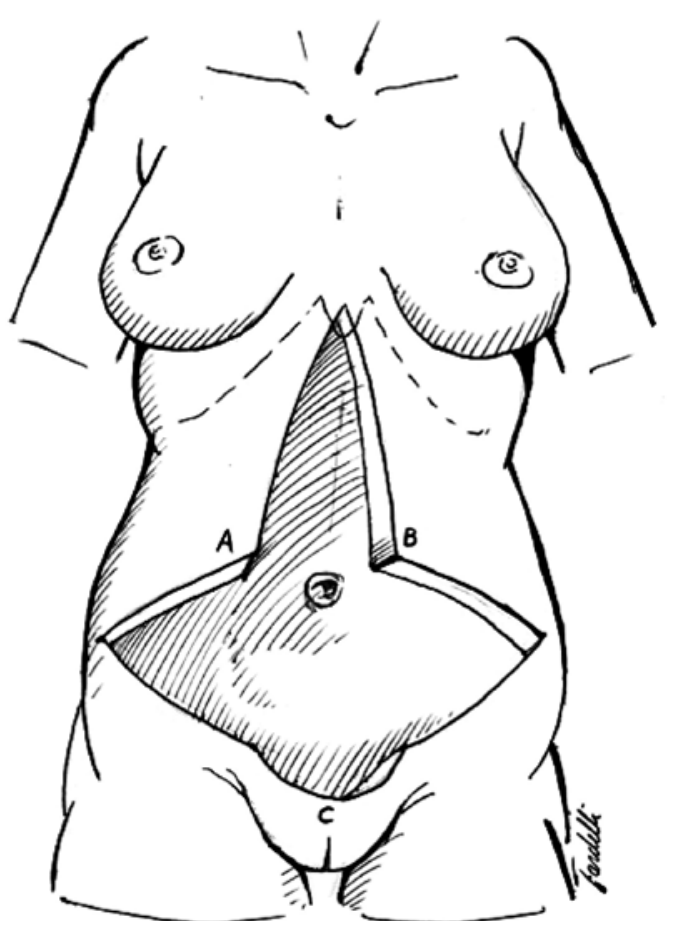

Figura 5 - Representação esquemática da marcação pré-operatória, evidenciando a exérese de semicírculo em região pubiana. 
caudalmente o excesso de pele para simular a posição final das cicatrizes e determinar a altura ideal da cicatriz umbilical. $\mathrm{O}$ ajuste final das dimensões definitivas dos retalhos, bem como da posição do neoumbigo, é agora definido.

\section{Cirurgia}

Inicia-se o procedimento com a infiltração das linhas de incisão pré-marcadas com solução fisiológica $(\mathrm{NaCl} 0,9 \%)$ contendo adrenalina diluída na concentração de 1:500.000 (com o intuito de reduzir o sangramento local) (Figura 6). Em seguida, realiza-se a ressecção do fuso de pele mediana até o plano aponeurótico (Figura 7), seguida pela plicatura do excesso de aponeurose do músculo reto do abdome ao longo da linha média com pontos em "X" de Nylon 2.0 (Figura 8). Neste passo, são também tratadas hérnias incisionais, quando presentes.

Procede-se, então, ao fechamento de toda a cicatriz vertical (Figura 9) (exceto na área da neo-onfaloplastia, como explicado a seguir). Após, incisa-se na marcação inferior, com subsequente descolamento supra-aponeurótico dos retalhos até atingir a área já descolada da incisão vertical. Os retalhos são então tracionados caudalmente, e o excesso cutâneo marcado com azul de metileno e ressecado (Figura 10), sendo realizada a sutura por planos da cicatriz inferior (Figura 11).

Quanto à cicatriz umbilical, adota-se a técnica de neoonfaloplastia semelhante àquela descrita por Franco et al.,10

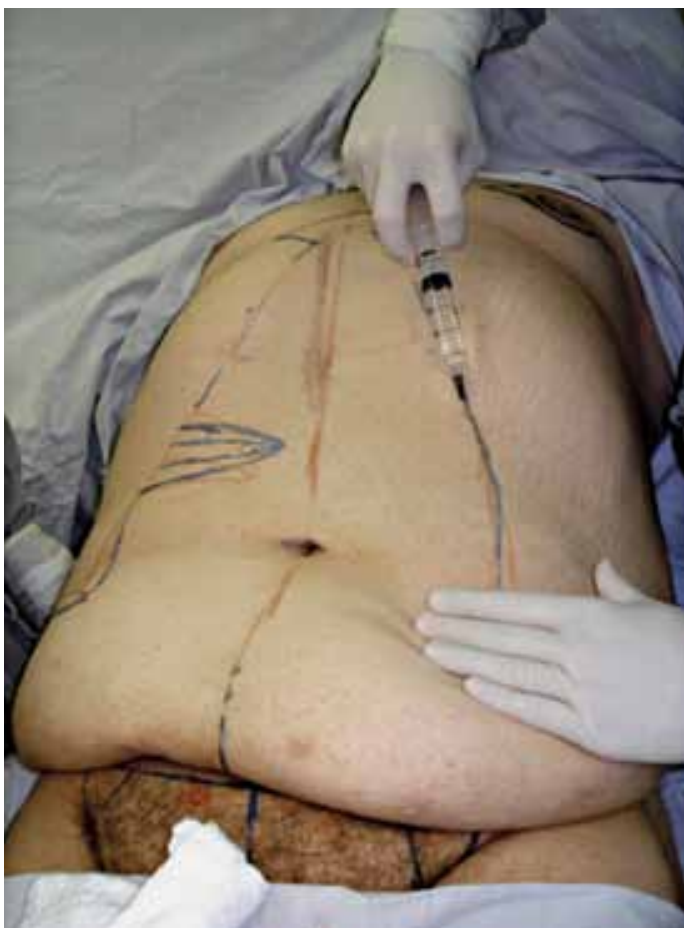

Figura 6 - Infiltração das incisões com solução de adrenalina 1:500.000.

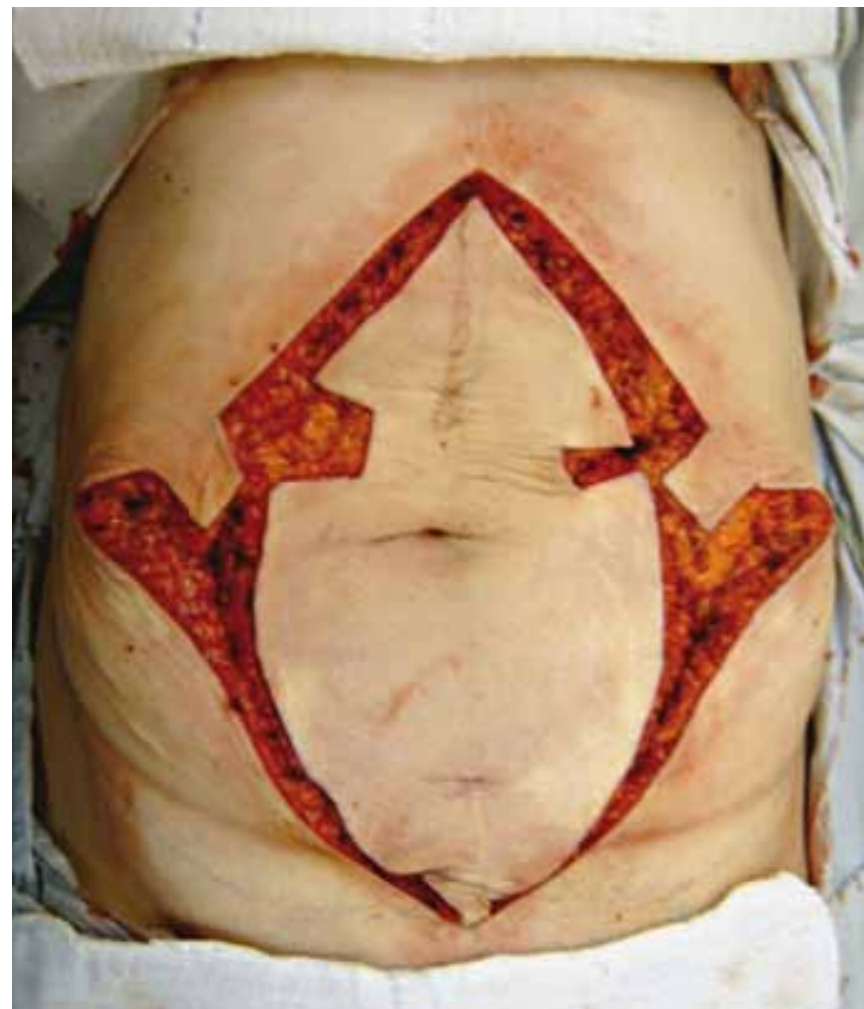

Figura 7 -Ressecção de fuso de pele mediana. Notar os pequenos retalhos laterais que formarão o neoumbigo.

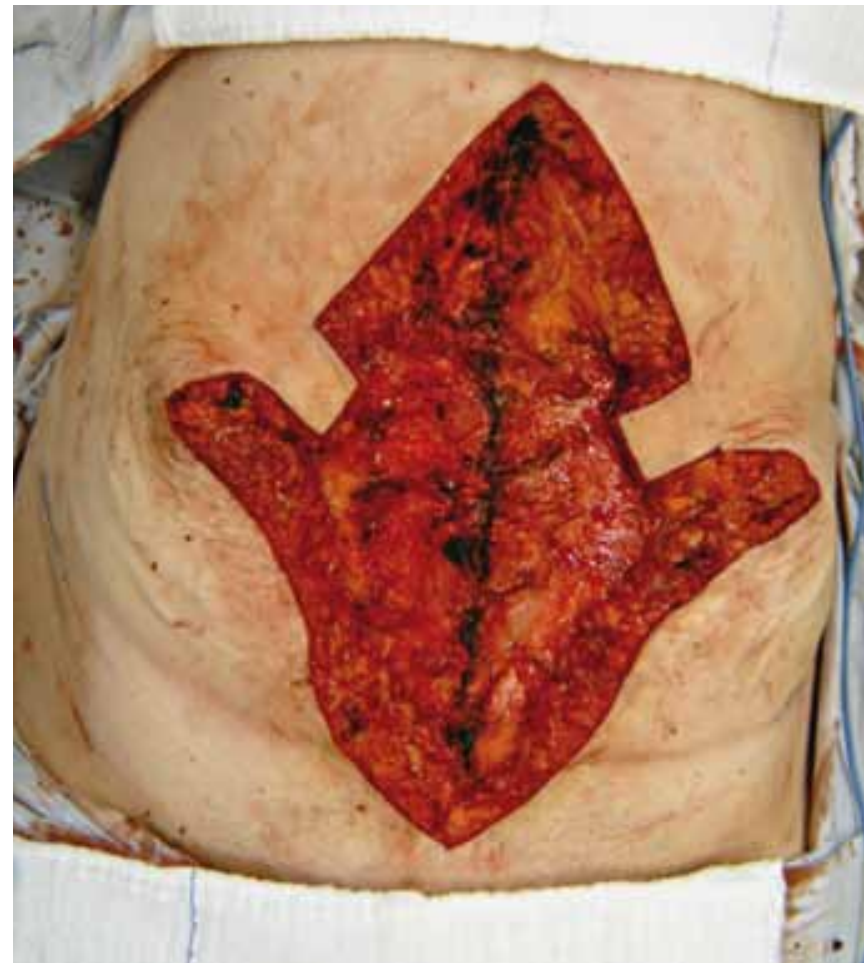

Figura 8 - Plicatura da aponeurose do músculo reto do abdome. 


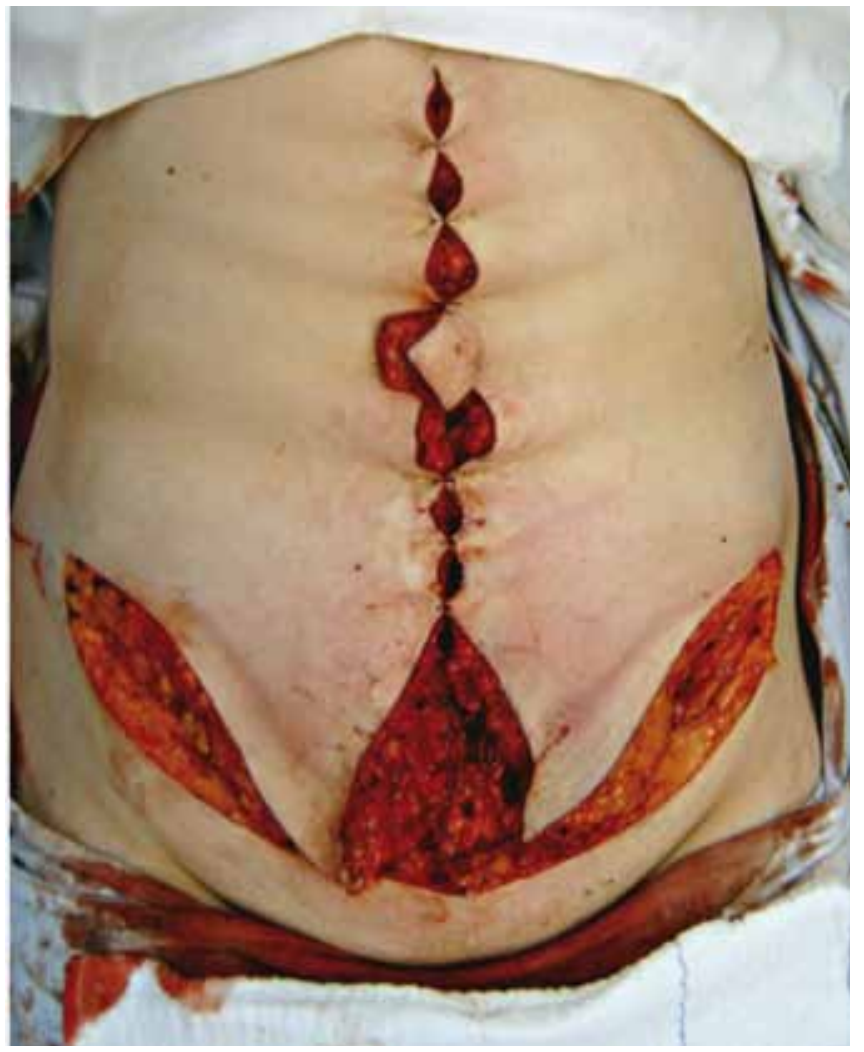

Figura 9 - Fechamento da cicatriz vertical.

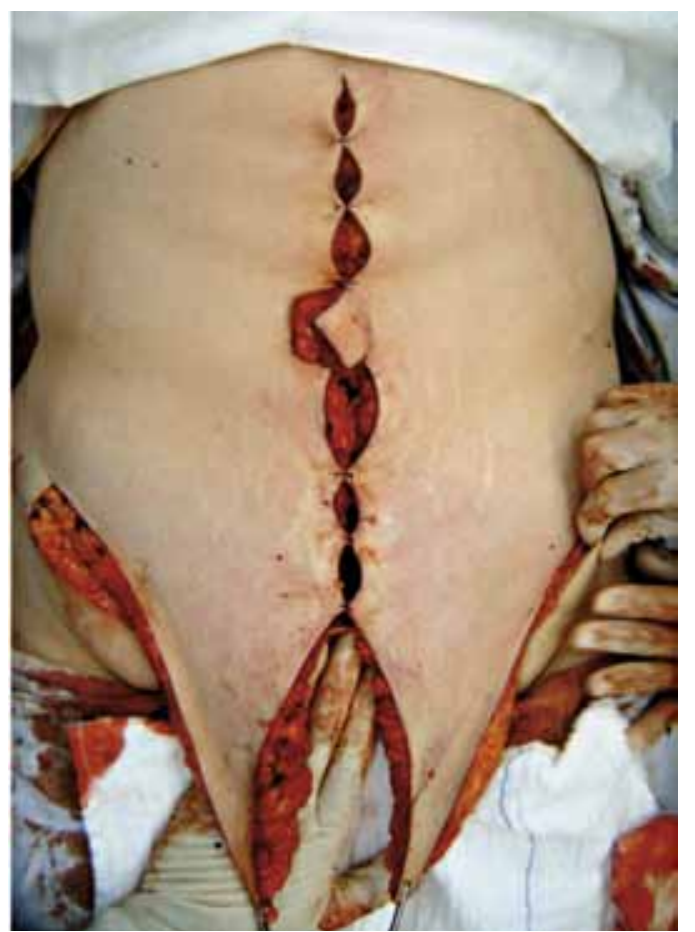

Figura 10 - Tração caudal dos retalhos para determinar o montante de ressecção cutânea horizontal.

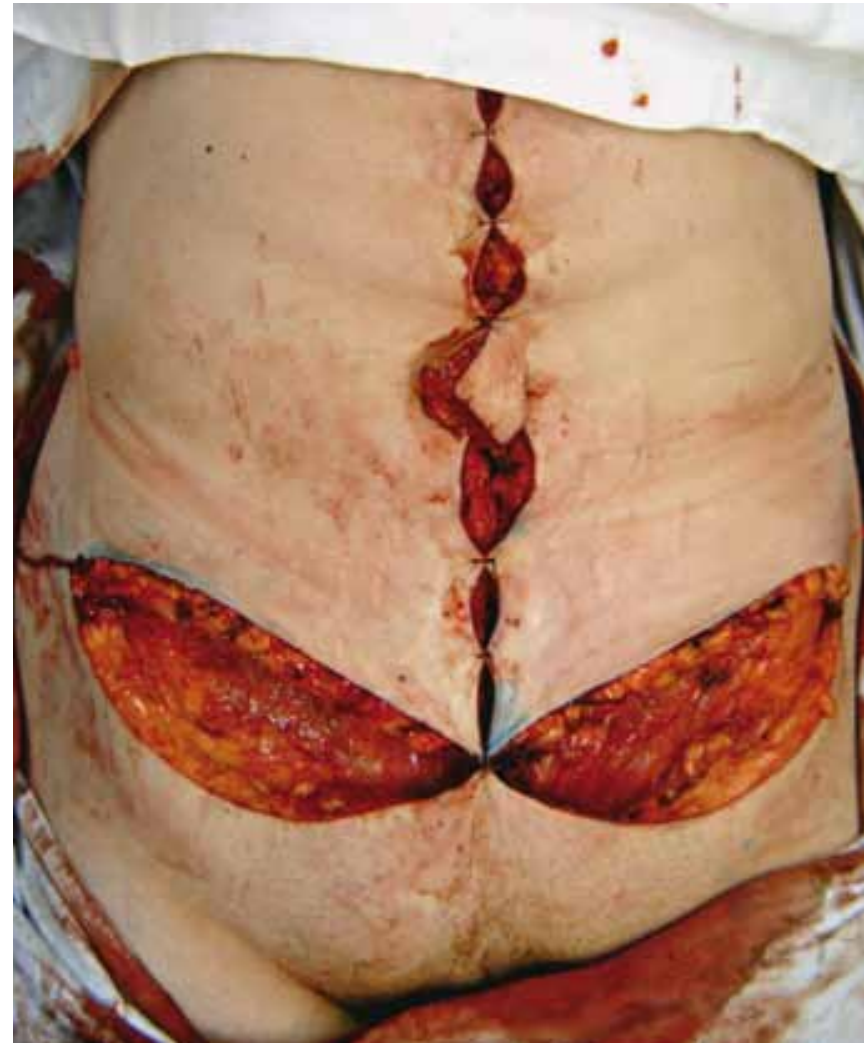

Figura 11 - Início da sutura da incisão horizontal.

e El-Dessouki ${ }^{11}$, entre outros autores ${ }^{12}$, empregando retalhos arqueados cutâneos nas bordas dos retalhos laterais (de aproximadamente $2 \mathrm{~cm}$ de largura e 2,5 cm de comprimento), ou outra técnica semelhante que emprega apenas um retalho, descrita por Pfulg et al. ${ }^{13}$. O excesso de pele e tecido subcutâneo dos retalhos previamente reservados durante a ressecção vertical é agora removido. Isto permite a confecção destes pequenos retalhos cutâneos definitivos que são fixados à aponeurose e entre si, formando uma nova cicatriz umbilical mais natural (Figura 12), no caso da primeira técnica e, no caso da segunda, o retalho único é rodado sobre si mesmo (Figura 13).

Para o tratamento do excesso de pele pubiana, bem como do excesso lateral do retalho inferior, adota-se ressecção de dois fusos de pele nas laterais do púbis, junto à prega inguinal bilateralmente ${ }^{14,15}$ (Figura 14), sendo a largura destes retalhos determinada por pregueamento manual durante a cirurgia.

Em todos os pacientes, foi realizada antibioticoprofilaxia na indução anestésica, com dois gramas de cefazolina. A profilaxia de trombose venosa profunda foi feita mediante enfaixamento compressivo das pernas, deambulação precoce no pós-operatório e uso de heparina sódica de baixo peso molecular subcutânea (Clexane ${ }^{\circledR}$ - 40 mg/dia subcutâneo por 10 dias) quando os pacientes apresentavam critérios para tal (segundo as normas de orientação clínica para a prevenção 


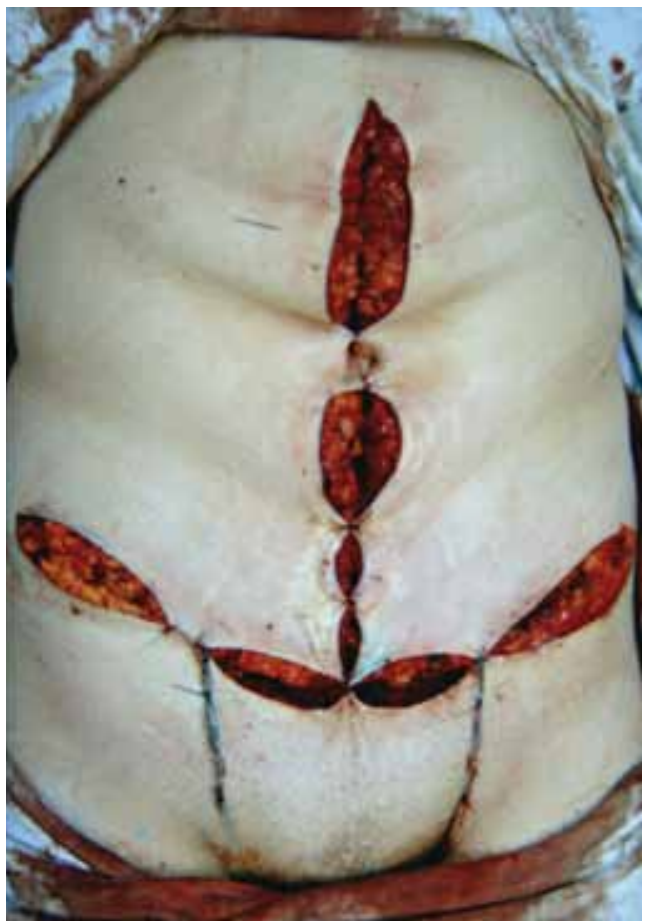

Figura 12 - Realização da neo-onfaloplastia através da sutura dos retalhos entre si e à aponeurose.

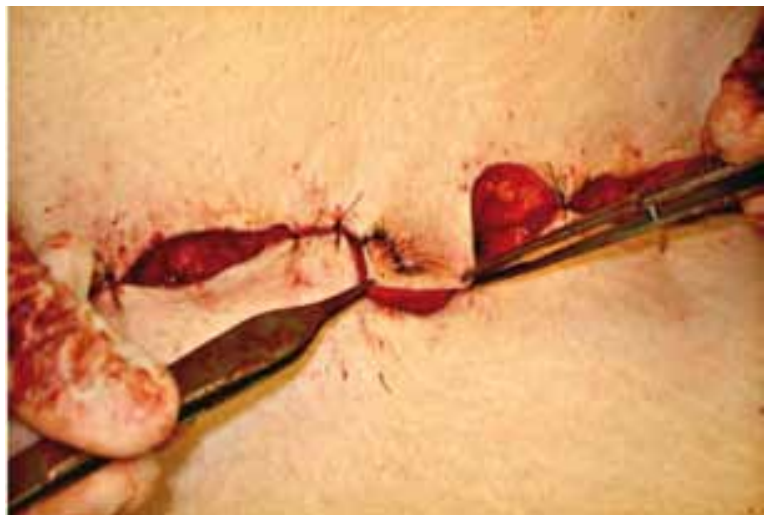

Figura 13 - Técnica alternativa de neo-onfaloplastia com uso de retalho único rodado sobre si mesmo.

da trombose venosa profunda da Sociedade Brasileira de Angiologia e Cirurgia Vascular ${ }^{16}$ ).

Em todos os casos, foi empregada drenagem fechada a vácuo com dreno tipo Portovac ${ }^{\circledR} 6.2$, mantido na média por 8,7 dias (3 a 20 dias).

\section{RESULTADOS}

O tempo cirúrgico médio foi de 199 minutos (115 a 410 minutos). $O$ peso das peças operatórias variou de $850 \mathrm{a} 7500 \mathrm{~g}$,

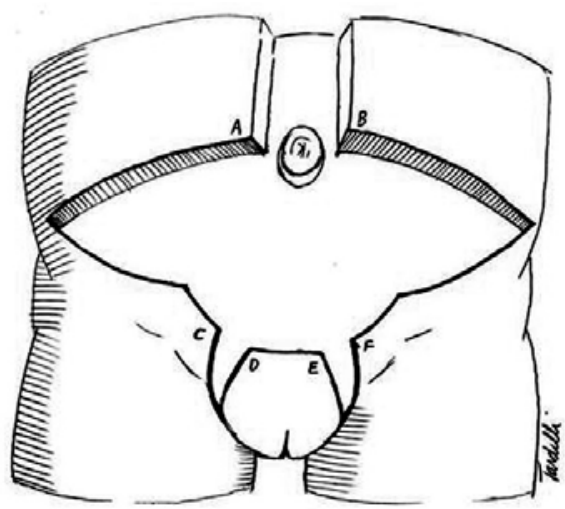

Figura 14 - Marcação da dermolipectomia pubiana com ressecção de dois fusos de pele laterais, conforme proposto por Arantes \& Flores $^{14}$.

com média de 2611 g. O tempo de internação habitual foi de 3 dias (média de 3,4 dias, variando de 2 a 15 dias), com exceção da paciente que apresentou hematoma volumoso, e de outra, que desenvolveu trombose venosa profunda, que permaneceram internadas por 12 e 15 dias, respectivamente.

As mudanças introduzidas na técnica resultaram em melhor posicionamento da cicatriz horizontal junto à região suprapúbica e melhor formato da mesma, com concavidade superior ao invés de inferior; devido à menor tensão final na intersecção das cicatrizes (Figuras 15 a 18). O neo-umbigo

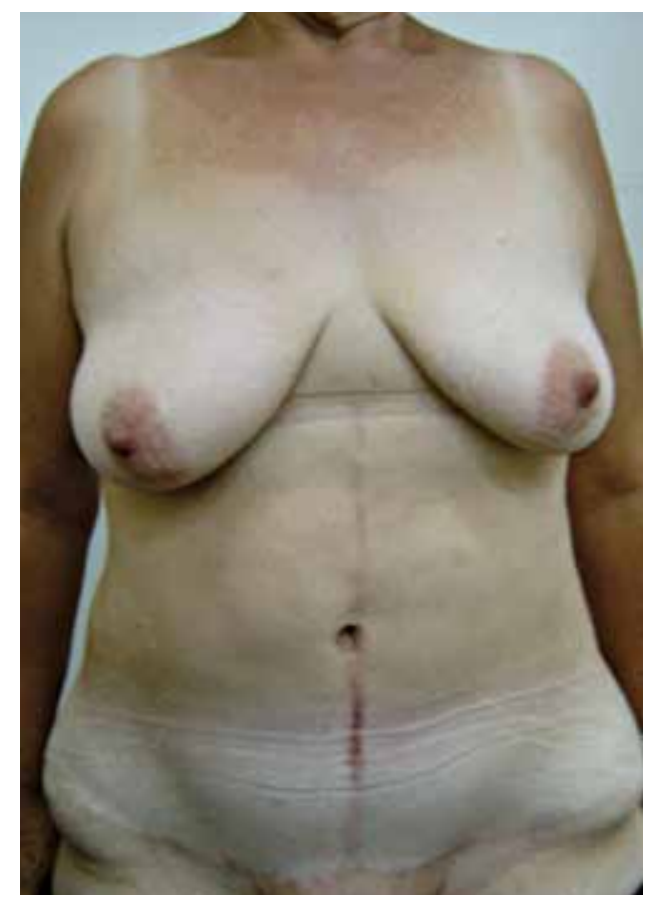

Figura 15 - Pós-operatório de seis meses demonstrando melhor posicionamento da cicatriz horizontal e umbigo com bom aspecto (neo-onfaloplastia com dois retalhos). 

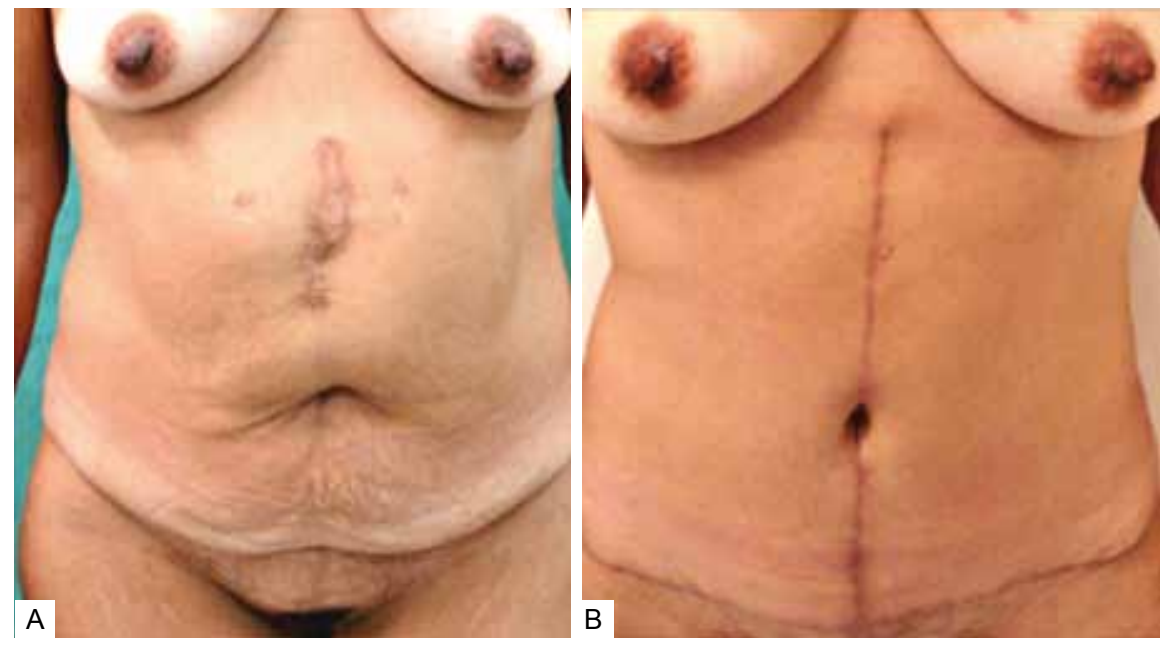

Figura 16 - Pré e pós-operatório de quatro meses (neoumbigo de retalho duplo).

também se mostrou mais natural na opinião da equipe e dos pacientes (Figuras 19 e 20), não havendo casos de estenose ou dilatação da cicatriz umbilical, como às vezes ocorria com a manutenção da cicatriz original.

As complicações apresentadas pelos pacientes foram: hematomas em 6 (8,9\%) casos, sendo que um deles necessitou de nova intervenção cirúrgica para drenagem (paciente portadora da Síndrome do Anti-Fosfolípide e em uso de Clexane ${ }^{\circledR}$

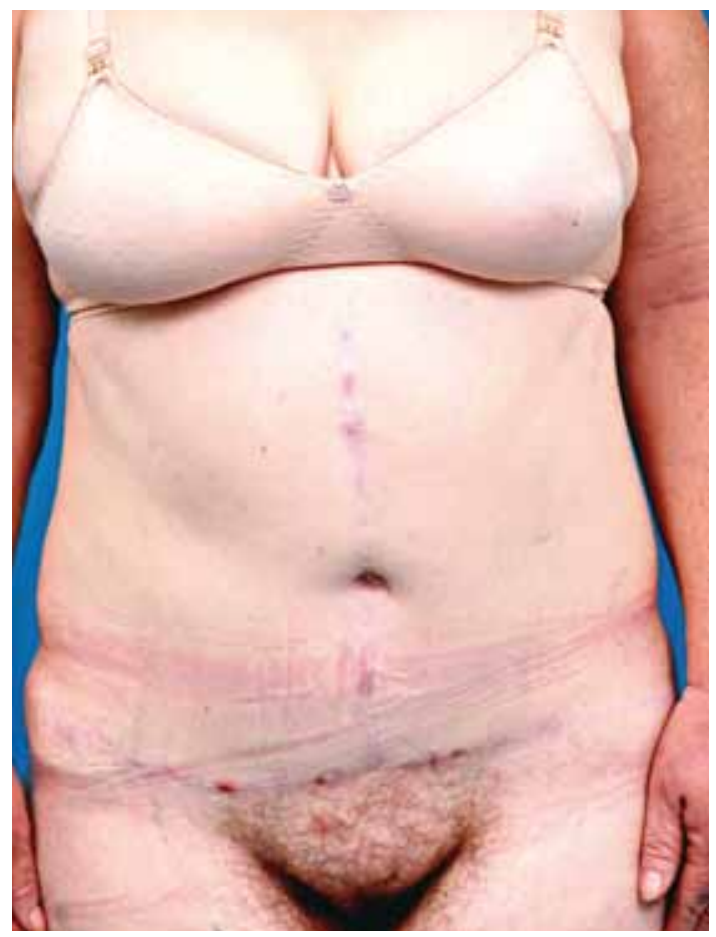

Figura 17 - Pós-operatório de seis meses (neo-onfaloplastia com dois retalhos). profilático); deiscências extensas de ferida operatória necessitando de ressutura em 5 (7,5\%) casos (sendo um deles a mesma paciente com Síndrome do Anti-Fosfolípide); seroma em $18(26,9 \%)$ casos; infecção de ferida operatória em 4 (6\%); granuloma de corpo estranho em 3 (4,5\%). Não houve nenhum caso de necrose, mesmo que parcial, dos retalhos. Houve um caso de trombose venosa profunda confirmada por ecodoppler e tratada com heparinização e repouso no leito.

\section{DISCUSSÃO}

Quando do início deste trabalho, em julho de 2006, as dermolipectomias abdominais clássicas nos pacientes pós-bariátricos eram realizadas da seguinte maneira: marcação do excesso de pele com o paciente em pé, através de prega bimanual do mesmo, sendo realizada a sua retirada em bloco (do componente vertical junto com o horizontal) na cirurgia, seguida de tratamento de herniações, quando necessário, drenagem da área cruenta com dreno a vácuo e fechamento primário sem descolamento dos retalhos com preservação da cicatriz umbilical, que era então exteriorizada no ramo vertical da cicatriz em âncora. O uso desta técnica de abdominoplastia em âncora resultava, na experiência dos autores, em algumas insatisfações que foram sanadas com as alterações aqui descritas.

Com esta nova abordagem, obtiveram-se resultados estéticos mais favoráveis e satisfatórios, com significante melhoria dos itens previamente relacionados, ou seja, melhor posicionamento e formato (concavidade superior e não inferior) da cicatriz horizontal, obtenção de distância adequada entre o neoumbigo e a cicatriz horizontal, redução da tensão na intersecção das cicatrizes, melhora importante do aspecto estético da nova cicatriz umbilical, correção efetiva do excesso de pele em região pubiana e na porção inferior da cicatriz horizontal (Figuras 15 a 20). 

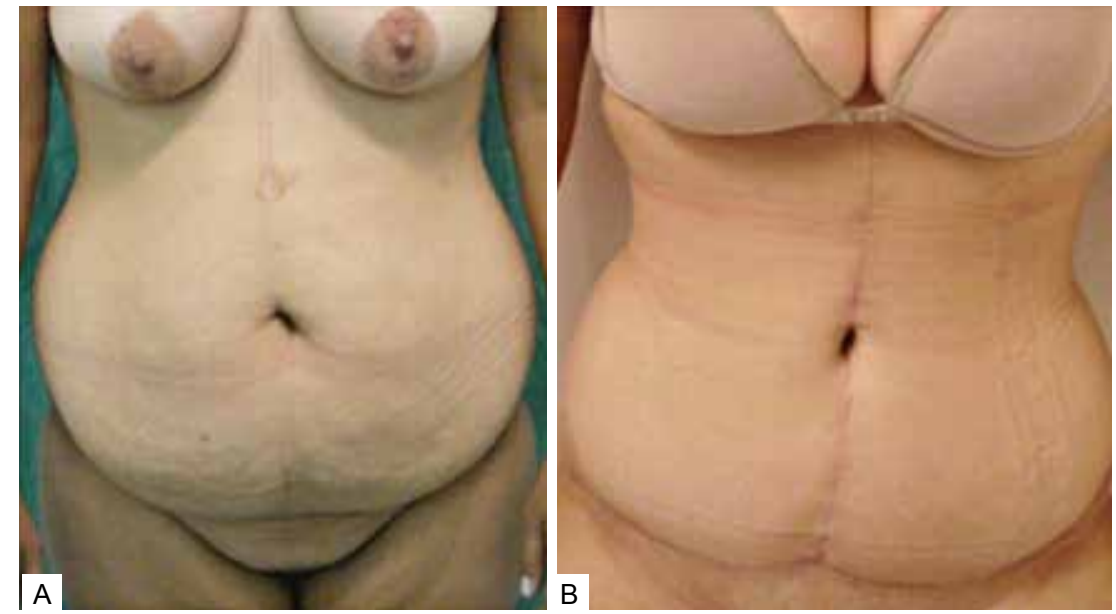

Figura 18 - Pré e pós-operatório de cinco meses (neo-onfaloplastia com retalho duplo).
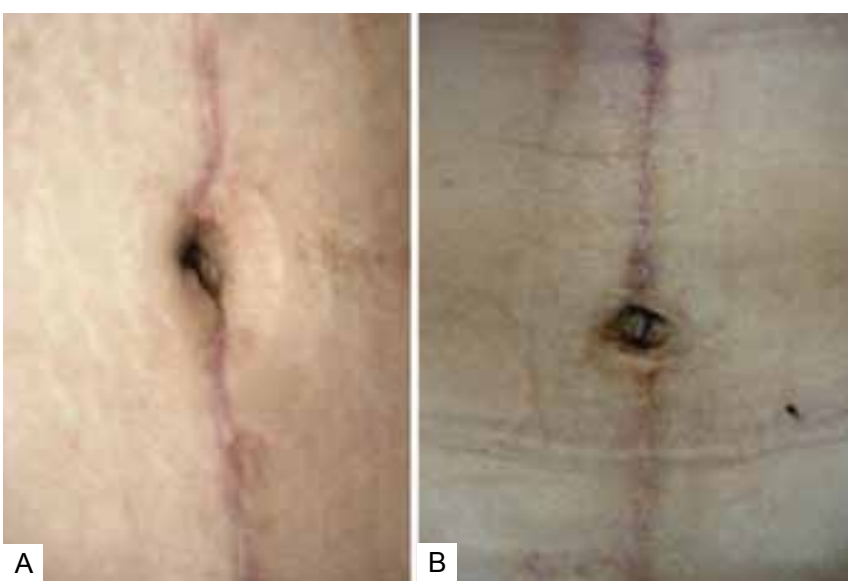

Figura 19 - Detalhe evidenciando o aspecto final de neoumbigos com a técnica de dois retalhos.

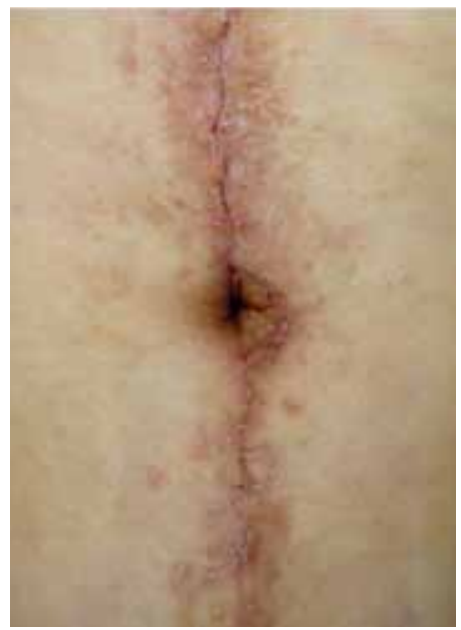

Figura 20 - Detalhe evidenciando o aspecto final de neoumbigo com a técnica de retalho único.
A adoção desta nova padronização não alterou o tempo cirúrgico em relação aos casos iniciais, em que se utilizou a técnica clássica,e nem o índice de complicações, exceto pela tendência à redução dos casos de deiscências pequenas principalmente na área de intersecção das cicatrizes.

A abordagem acima descrita evoluiu ao longo destes 67 casos com maior satisfação da equipe e dos pacientes, quesito no qual a neo-onfaloplastia é emblemática, pois, no início, ela era realizada apenas naqueles pacientes em que o umbigo original apresentavase impossibilitado de ser preservado por se localizar sobre sacos herniários volumosos, prejudicando sua viabilidade; mas a partir destes casos, outros pacientes passaram a questionar a equipe sobre a possibilidade de realizar o mesmo procedimento (mesmo quando a cicatriz umbilical original podia ser preservada), por considerarem mais estéticos os resultados que viram em colegas de ambulatório, sendo que atualmente a totalidade dos pacientes opta por esta modalidade quando confrontada com a opção ilustrada por fotos de pré e pós-operatório.

A maior dificuldade encontrada com relação à neo-onfaloplastia reside em determinar a altura adequada para se desenhar os retalhos, de tal forma que se localizem na altura da linha que une as cristas ilíacas ântero-superiores, ao fim do procedimento. Para contornar esta dificuldade, marcam-se inicialmente os retalhos com aproximadamente 6 a $7 \mathrm{~cm}$ de largura, fornecendo margem de manobra para que, quando as suturas já estejam em suas posições finais, possa-se determinar apuradamente a altura ideal para a nova cicatriz umbilical, desprezando o excesso de tecido, seja na porção superior ou inferior dos retalhos, conservando a parte do retalho que esteja na altura desejada.

\section{CONCLUSÃO}

A adoção das alterações táticas descritas na padronização das abdominoplastias em âncora permitiu minorar as 
insatisfações previamente descritas com a técnica clássica, principalmente reduzindo a tensão nas cicatrizes e melhorando seu posicionamento final.

Este trabalho mostra a experiência dos autores e a sua evolução neste procedimento, alterando a sistematização e buscando refinar os resultados obtidos, corrigindo os pontos inadequados observados no seguimento destes pacientes.

\section{REFERÊNCIAS}

1. Bozola AR, Bozola AC. Abdominoplastias. In: Mélega JM, ed. Cirurgia plástica: fundamentos e arte - cirurgia estética. Rio de Janeiro:Medsi;2003. p.609-23.

2. Carreirão S, Pereira L. Planejamento e indicações técnicas em abdominoplastia. In: Resende JHC, ed. Tratado de cirurgia plástica na obesidade. Rio de Janeiro:Rubio;2008. p.275-83.

3. Resende JHC. Tratamento cirúrgico complementar na obesidade mórbida. In: Mélega JM, ed. Cirurgia plástica: fundamentos e arte - cirurgia estética. Rio de Janeiro:Medsi;2003. p.687-94.

4. Borud LJ, Warren AG. Modified vertical abdominoplasty in the massive weight loss patient. Plast Reconstr Surg. 2007;119(6):1911-21.

5. Roxo CP, Magalhães FL, Alcântara BJC, Roxo ACW. Abdominoplastia em âncora. In: Resende JHC, ed. Tratado de cirurgia plástica na obesidade. Rio de Janeiro:Rubio;2008. p.291-302.
6. Fraccalvieri M, Datta G, Bogetti P, Verna G, Pedrale R, Bocchiotti MA, et al. Abdominoplasty after weight loss in morbidly obese patients: a 4-year clinical experience. Obes Surg. 2007;17(10):1319-24.

7. Fotopoulos L, Kehagias I, Kalfarentzos F. Dermolipectomies following weight loss after surgery for morbid obesity. Obes Surg. 2000;10(5):451-9.

8. Marking and operative techniques. Plast Reconstr Surg. 2006;117(1 Suppl):45S-73S

9. Franco T, Franco D. Neoomphaloplasty: an old and new technique. Aesthetic Plast Surg. 1999;23(2):151-4.

10. Franco D, Medeiros J, Farias C, Franco T. Umbilical reconstruction for patients with a midline scar. Aesthetic Plast Surg. 2006;30(5):595-8.

11. el-Dessouki NI, Shehata SM, Torki AM, Hashish AA. Double half-cone flap umbilicoplasty: a new technique for the proboscoid umbilical hernia in children. Hernia. 2004;8(3):182-5.

12. Silva FN, Oliveira EA. Neo-onfaloplastia na abdominoplastia vertical. Rev Bras Cir Plást. 2010;25(2):330-6.

13. Pfulg M, Van de Sijpe K, Blondeel P. A simple new technique for neoumbilicoplasty. Br J Plast Surg. 2005;58(5):688-91.

14. Arantes HL, Flores LR. Dermolipectomia pubiana pós-cirurgia bariátrica e severa perda ponderal. In: XXXIX Congresso Brasileiro de Cirurgia Plástica; 2002 Nov 20-23; Salvador, Brasil.

15. Tavares Filho JM, Belerique M, Franco D, Franco T. Dermolipectomy of the pubic area associated with abdominoplasty. Aesthetic Plast Surg. 2007;31(1):12-5.

16. Maffei FHA, Caiafa JS, Ramacciotti E, Castro AA para o Grupo de Elaboração de Normas de OrientaçãoClínica em Trombose Venosa Profunda da SBACV. Normas de orientação clínica para a prevenção, o diagnóstico e o tratamento da trombose venosa profunda. J Vasc Br. 2005;4(Supl.3):S205-20.

\section{Correspondência para:}

Daniel Bacco Vilela

Hospital das Clínicas - FMRP-USP

Av. Bandeirantes, 3900 - Monte Alegre - Ribeirão Preto, SP, Brasil - CEP: 14040-140

E-mail: danielbvilela@gmail.com 